\title{
In-situ tensile observation of deformation twin in TWIP steel using TEM
}

Sung-Il Baik, ${ }^{*}$ Tae-Young Ahn, ${ }^{*}$ Woong-Pyo Hong, ${ }^{*}$ Yeon-Seung Jung, ${ }^{* *}$ Young-Kook Lee, ${ }^{* *}$ and Young-Woon Kim.*

* Department of Materials Science and Engineering, Seoul National University, Gwanak-gu Silimdong San 56-1, Seoul 151-744, Korea

** Department of Metallurgical Engineering, Yonsei University, Shinchon-dong 134, Seodaemun-gu, Seoul 120-749, Korea

Fe-Mn twinning-induced plasticity (TWIP) steels have attracted research attention due to its high strength and exceptional plasticity in recent years [1]. These enhanced superior formability and strength satisfied the improved safety standards for high energy absorption in the construction and crash resistance in the automotive industry. In this Fe - Mn TWIP steel, stacking fault energy (SFE) is the key parameter to determine the strengthening mechanism [2]. But stacking fault related microstructure changes were well studied but the formation mechanism of twin is not well understood. In this study, the generation of mechanical twin was observed by in-situ tensile test in TEM and the effect of SFE was discussed linked with the measured SFE.

Plan view TEM (PTEM) tensile samples were prepared by wire-cutting method. Sample thinning was made by going through a typical sample preparation technique by grinding polishing, and followed by electrochemical thinning. Philips CM20 and JEOL 3000F were used to investigate the microstructural changes after in-situ deformation.

Figure 1 shows the sequential view in in-situ experiment for the generation of dislocations movement, planar glide, stacking fault, twin at near [110] zone axis of TWIP steel. True strain in tensile sample was marked in the bottom, which was measured by reference point marked before tensile strain. Dislocation movement in this TWIP steel starts at (111) planar slip plane and then split into partial dislocations to form stacking faults (SF). Next Crossed dislocation generate across the planar glide. Sequential SF generate twin along the crossed dislocation. Twin acts as a fracture source in the further strain along the (111) plane. Figure 2 are post- mortem observation of tensile area from figure 1(f). Stacking fault and twin in TWIP steel are shown in high resolution image and selected area diffraction pattern at [110] zone axis. Darkfield image of (002) twin spot from SADP confirmed that there existed twin (Fig 2 (a)). In the initial stage, planar glide occurred and formed stacking faults with further strain, which was, in turn, believed to form twins. This movement of dislocation and twin generation was affected by the weight percent of Mn contents. Reduction of Mn contents decreased of stacking fault energy. And these lowering of SFE lead to non-sequential stacking fault and makes epsilon phase.

\section{References}

[1] O. Grassel et. al., J. Appl. Phys., IV 5, (1997) 383.

[2] B. X. Huang et. al., Meter. Sci. Eng. A 438-440 (2006) 306.

[3] This study was supported by a grant from the Fundamental R\&D Program for Core Technology of Materials funded by the Ministry of Commerce, Industry and Energy, Republic of Korea. 

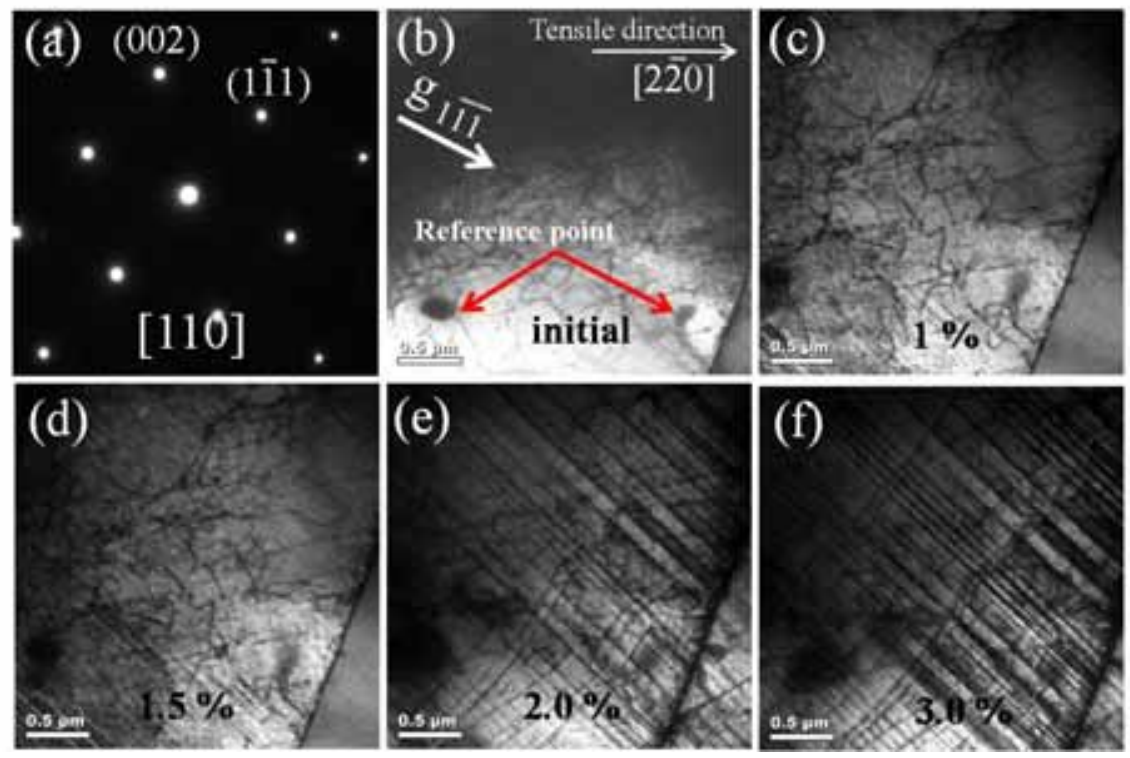

Figure 1 In-situ tensile experiment in TWIP steel. (a) Selected area diffraction Pattern(SADP) (b) Initial tensile stage. (c) Before the generation of planar glide. (d) After the generation of planar glide. (e) Dislocation generation across the planar glide. (f) Twin generation along the crossed dislocation.
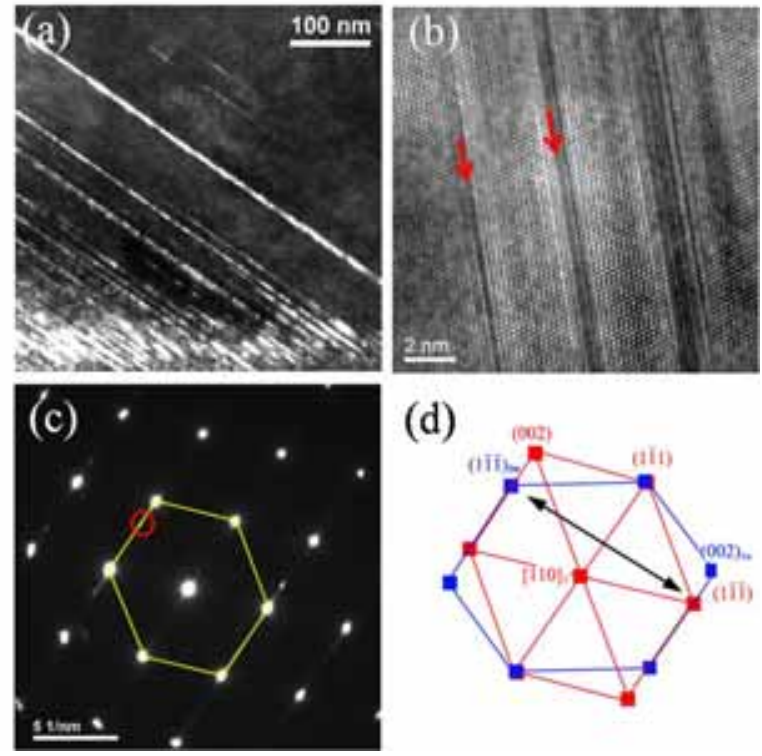

Figure 2 Post- mortem observation of strained area from figure 1. (a) Darkfield image from (002) $\mathrm{m}$ plane. (b) High resolution image. (c) Selected area diffraction pattern (SADP) of (a). (d) Schematic view of SADP. 